\title{
Tumor Microenvironment and Obesity
}

\section{Bubnovskaya $^{1 *}$, I Ganusevich ${ }^{1}$ and D Osinsky ${ }^{2}$}

${ }^{1}$ RE Kavetsky Institute of Experimental Pathology, Oncology and Radiobiology,

National Academy of Sciences of Ukraine, Kyiv, Ukraine

${ }^{2}$ City Clinical Oncological Center, Kiev, Ukraine

*Corresponding Author: L Bubnovskaya, RE Kavetsky Institute of Experimental

Pathology, Oncology and Radiobiology, National Academy of Sciences of Ukraine, Kyiv, Ukraine.

The worldwide obesity epidemic is a growing public health problem. Obesity is associated with the development of many disorders such as insulin resistance, cardiovascular disease, diabetes, depression. Epidemiologic, clinical, and preclinical data suggest that various common types of cancer are affected by obesity and it was revealed a link between obesity and the more aggressive character of malignancy. Obesity is decided as an independent prognostic factor of recurrence and survival.

There is adequate evidence support an association between obesity and increased risk of developing colorectal, breast, gastric, glioma, endometrial, kidney, esophageal, liver, gallbladder, pancreatic, and thyroid, prostate cancers, as well as non-Hodgkin's lymphoma and myeloma especially those which grow in the anatomical vicinity of adipose tissue.

The excess of lipids from adipocytes in the tumor microenvironment is increasingly recognized as a growing cause leading to tumor progression and uncontrolled growth and negatively influences prognosis in several cancer types [1]. Excessive adipose expansion during obesity causes immune dysregulation as well chronic low-grade inflammation. Obesity-induced inflammation known as mediator of cancer promotes the genotoxic stress thereby may influence tumor initiation and progression [2].

Tumor is more than insular masses of proliferating cancer cells (Gemma Texidó, 2013). It is complicated system of distinct cell types that are in heterotypic interactions with each other and surrounding tissue owing to the tumor microenvironment is formed.
Tumor microenvironment closely connected with the organism where it plays a pivotal role in the development and behavior of malignant neoplasia [3,4]. Growing body of evidence now points to an essential role of the tumor microenvironment as a critical regulator of tumor cell metastasis, which facilitates the homing and expansion of the tumor clone from the primary neoplasm to distant organs.

There was shown strong evidence that during cancer progression there is metabolic crosstalk between tumor cells and adjacent adipose tissue. Adipocytes supply cancer cells with adipokines and lipids and at the same time stromal and immune cells from adipose tissue secrete paracrine factors into the tumor microenvironment thereby infiltrate tumors [5].That is why a variety of solid tumors grow in close proximity with adipocytes and adipose-associated stromal and vascular components.

Mechanisms whereby adipose accumulation increases risk of tumor onset and/or mediates tumor progression in adipose-adjacent cancers are multifactorial, complex, and likely tissue/organspecific, in part due to unique paracrine and physical interactions occurring between cancer cells and adjacent adipose tissue developing the adverse metabolic milieu associated with obesity [6].

Obesity essentially influences metabolic and inflammatory changes in adipose tissue microenvironment by means of numerous ways including induction of fibrosis and angiogenesis, increased stem cell abundance, and expansion of proinflammatory immune cells. Excessive adipose expansion during obesity causes

Citation: L Bubnovskaya., et al. "Tumor Microenvironment and Obesity". Acta Scientific Cancer Biology 5.6 (2021): 05-08. 
adipose dysfunction which characterized by adipocyte hypertrophy, hypoxia, adipokine secretion that form a key component of the paracrine signaling in the tumor microenvironment, hormonal resistance and altered metabolic responses, together contributing to insulin resistance, metabolic syndrome, chronic disease and also related to chemotherapeutic resistance. These cells by their ability to secrete elevated levels of pro-inflammatory factors, extracellular matrix proteins and proteases, involved in their remodeling, as well as to release free fatty acid, modulate the tumor microenvironment by promoting angiogenesis, production of proinflammatory cytokines, affecting immune cells and altering metabolism. Adipocytes express a complex and highly adaptive biological profile being capable to selectively activate of key metabolic and oncogenic signaling pathways including Wnt/ $\beta$-catenin and Hippo/YAP signaling, that which may be an active local player in establishing the peritumoral environment promoting tumor growth and progression and can mediate local and systemic effects.

Expansion of adipose tissue during progression to obesity requires concomitant expansion of the adipose vascular bed through the process known as angiogenesis, the formation of new blood vessels from preexisting vessels. When expansion of the vasculature does not occur in proportion to the expansion of adipocyte volume (hypertrophy), regional hypoxia develops, resulting in activation of the transcriptional complex hypoxia-inducible factor 1 (HIF-1) through stabilization of the HIF-1 $\alpha$ subunit. HIF-1 mediates upregulation of inflammatory and proangiogenic signaling pathways in adipocytes, endothelial cells, and immune cells, induces vascular growth, facilitating further tissue expansion.

Peculiarities of tumor microenvironment under obesity may play definite role in $\mathrm{nCoV}$ invasion of host cells. It worth to note that patients with cancer have worse outcomes from COVID-19 and there is strong association between obesity and the severity of COVID-19 infection. Transmembrane glycoprotein (CD147), which plays fundamental roles in intercellular interactions in numerous pathological and physiological processes, has been recognized as one of essential receptor for novel pathway in $\mathrm{nCoV}$ invasion of host cells. It is highly expressed in various cancer cells with very limited expression in normal tissues [7.] Overexpressed CD147 orchestrates tumor cell proliferation, stimulates fibroblasts to produce a large number of matrix metalloproteinases leading to tumor cell invasion, plays pivotal roles in intercellular interactions involved in tumor metastasis, angiogenesis and tumor cell glycoly- sis through critical molecules such as monocarboxylate transporters, matrix metalloproteinases, especially under hypoxic conditions. Moreover elevated level of transmembrane serine protease (TMPRSS2) seeing in tumors is needed for priming of the spike (S) protein of the virus. Obesity alters TMPRSS2 as a host cell factor that is critical for spread of several clinically relevant viruses, including influenza A viruses and coronaviruses. That all promotes increasing risk of transmission of infection and management of patients with cancer especially having obesity has to be provided with more care [8].

Tumor hypoxia has been associated with the development of aggressive phenotypes in cancer cells and is a powerful, independent prognostic factor in multiple types of cancer. Excessive adipose expansion during obesity is a cause of hypoxia development and appears to be a key factor in adipose tissue dysfunction in obesity. Alteration of adipose tissue oxygenation promotes a favorable environment for tumor. Glycolytic reprogramming of tumor metabolism of cancer cells under tumor hypoxic microenvironment known as metabolic symbiosis facilitates enhancement the utilization of fuel sources of oxidative metabolism such as lactate, glutamine, and fatty acids released by surrounding cells, including other cancer cells and adipocytes [9].

Intertumoral hypoxia recognized as an independent marker from clinical size, stage, histology and grade but hypoxia is intensified by the hemoglobin level in anemic patients, especially in tumor (areas) with low perfusion rates. Experimental and clinical studies testify that pretreatment anemia is associated with highly hypoxic tumors [10]. Even mild anemia may be as a major causative factor for the development of hypoxia or anoxia.

Hypoxia as well anemia due to worsening of tumor hypoxia especially under excessive adiposity can have a profound impact on physical and psychosocial function and may promote metastasis and cause resistance to treatment, as they make solid tumors resistant to ionizing radiation and some forms of chemotherapy. By means of meta-analysis it was shown that pretreatment anemia plays significant role in decreasing long-term overall survival and disease-free survival of patients.

Appropriate correction of anemia status may at least partially help to overcome the inherent resistance to treatment in anemic patients and to some extent to suppose definite control under tu- 
mor oxygenation resulting in better overall survival of patients [10-12].

Not excepting that adipose tissue microenvironment may pave the way for understanding such an unique phenomenon that was dubbed as "obesity paradox". It is known that obesity as established cause of renal cell cancer, is associated with improved outcomes of these patients and moreover even with a better prognosis than for patients with a normal weight. $[13,14]$.

Unexpected better survival in obese patients with gastric cancer also was shown. Moreover it was detected significantly decreased frequency of disseminated tumor cells in bone marrow under obesity. The receptivity of the bone marrow milieu and its components is essential for tumor cells that preferentially metastasize to the bones, as it reported for breast, prostate, and lung cancer. Most probably that excess of tumor adipocites may play a potentially critical role in the regulation of metabolic functions and create specific metabolic changes in tumor microenvironment to prevent tumor cells escaping from the primary lesion but molecular mechanisms are still poorly understood. It may be supposed that survival of patients is influenced mainly by high adiposity of tumors and such influence depends essentially on the BMI [15]. The same situation have been observed in patients with other disorders, such as heart disease, diabetes and even hip fractures.

The data are very controversial and the real question is whether the "obesity paradox" is a true biological phenomenon or an artefact of how studies are conducted, says Martin Lajous, an epidemiologist at Harvard, "obese people have a higher incidence of kidney cancer, but are also more likely to survive the disease" [13]. Most probability that high adiposity contributed to tumor but this a phenomenon could be explained away by other confounding factors such as variety of environmental factors or genetic predisposition. "Obesity appears to be protective", Banack from McGill University in Montreal says, "but that's an illusion, more likely is that the disease variants that come from other causes are so much worse" [13].

Patients with cancer and obesity which represent a particularly vulnerable population are in need of special attention and care. The hope remains for developing more precise cancer therapy altering metabolic pathways seen in tumor cells thus represent new targets for potential treatment specific to a person's cancer subtype, as well as to provide better prognoses to such unique patients that it will be emerged as beneficial.

\section{Conflict of Interest Statement}

The authors declare that they have not conflict of interest.

\section{Bibliography}

1. Isao Otsuka. "Cutaneous Metastases in Ovarian Cancer". Cancers 11.9 (2019): 1292.

2. I Stuart Wood., et al. "Cellular hypoxia and adipose tissue dysfunction in obesity". Proceedings of the Nutrition Society 68.4 (2009): 370-377.

3. Gemma Texidó. "Genetically engineered animal models for in vivo target identification and validation in oncology". Methods in Molecular Biology 986 (2013): 281-305.

4. Hanahan D and Weinberg R. "Hallmarks of cancer: the Next generation". Cell 144.5 (2011): 646-674.

5. Ernst Lengye., et al. "Cancer as a Matter of Fat: The Crosstalk between Adipose Tissue and Tumors". Trends Cancer 4.5 (2018): 374-384.

6. Ubaldo E Martinez-Outschoorn., et al. "Power Surge: Supporting Cells "Fuel" Cancer Cell Mitochondria". Cell Metabolism 15.1 (2012): 4-5.

7. Li Yan., et al. "Roles of the multifunctional glycoprotein, EMMPRIN (basigin; CD147), in tumour progression". Thrombosis and Haemostasis 93.2 (2005): 199-204.

8. Dylan C Sarver and G William Wong. "Obesity alters Ace 2 and Tmprss 2 expression in lung, trachea, and esophagus in a sexdependent manner: Implications for COVID-19". Biochemical and Biophysical Research Communications 538 (2021): 92-96.

9. Ana I Pérez-Hernández., et al. "Mechanisms linking excess adiposity and carcinogenesis promotion expands". Frontiers in Endocrinology 5 (2014): 65.

10. P Vaupel., et al. "Hypoxia in breast cancer: role of blood flow, oxygen diffusion distances, and anemia in the development of oxygen depletion". Advances in Experimental Medicine and Biology 566 (2005): 333-342.

11. Peter Vaupel., et al. "Impact of Hemoglobin Levels on Tumor Oxygenation: the Higher, the Better?". Strahlentherapie und nkologie 182.2 (2006): 63-71. 
12. Chiara Braconi Mario Scartozzi. Anemia may influence the outcome of patients undergoing neo-adjuvant treatment of rectal cancer". Annals of Oncology 17.11 (2006): 1661-1664.

13. Sujata Gupta. “Obesity: The fat advantage”. Nature 537 (2016) S100-122.

14. Helena Furberg., et al. "Transcriptomic signatures related to the obesity paradox in patients with clear cell renal cell carcinoma: a cohort study". The Lancet Oncology 21.2 (2020): 283293.

15. L Bubnovskaya., et al. "Disseminated tumor cells in bone marrow in gastric cancer patients with obesity". Journal of Cancer Research and Therapeutics 4.5 (2019): 1-3.

\section{Assets from publication with us}

- Prompt Acknowledgement after receiving the article

- Thorough Double blinded peer review

- Rapid Publication

- Issue of Publication Certificate

- High visibility of your Published work

Website: www.actascientific.com/

Submit Article: www.actascientific.com/submission.php

Email us: editor@actascientific.com

Contact us: +919182824667 